\title{
Der fachdidaktische Grundkonsens 2.0 in der Verbundregion Nordost. Perspektiven einer zukunftsfähigen Orientierungs- hilfe im GW-Unterricht
}

\author{
herbert.pichler@univie.ac.at, Arbeitsgruppe Fachdidaktik Geographie und wirtschaftliche Bildung, Institut für Geographie und Regio- \\ nalforschung, Universität Wien \\ ** christian.fridrich@phwien.ac.at, Fachbereich Geographische und Sozioökonomische Bildung, Pädagogische Hochschule Wien \\ *** christian.vielhaber@univie.ac.at, Arbeitsgruppe Fachdidaktik Geographie und wirtschaftliche Bildung, Institut für Geographie und \\ Regionalforschung, Universität Wien \\ ${ }^{* * * *}$ felix.magnus.bergmeister@univie.ac.at, Arbeitsgruppe Fachdidaktik Geographie und wirtschaftliche Bildung, Institut für Geographie \\ und Regionalforschung, Universität Wien
}

Keywords: fachdidaktische Bildung, Handlungs- und Begründungsfähigkeit, Mehrperspektivität, Lehrer/innenbildung, didaktische Modelle und Konzepte, didaktisch und methodische Prinzipien, geographische und ökonomische Basiskonzepte

Aus Anlass der Vernetzung der Lehrer/innenbildung in der Verbundregion Nordost zwischen der Universität Wien, der Pädagogischen Hochschule Wien, der Pädagogischen Hochschule Niederösterreich und der Kirchlichen Pädagogischen Hochschule Wien/Krems sowie anlässlich des anstehenden Jubiläums 55 Jahre Geographie und Wirtschaftskunde haben wir es uns zur Aufgabe gemacht, den Fachdidaktischen Grundkonsens (Pichler \& Vielhaber 2012, 45 f.) gemeinsam zu überarbeiten. Alle Lehrenden der Fachdidaktik GW der Verbundregion Nordost waren eingeladen, in einem partizipatorischen Diskussions- und Schreibprozess ihre Erfahrungen aus der Lehrpraxis und daraus hervorgehende Anregungen in die Neukonzeption der Eckpfeiler der fachdidaktischen Bildung einfließen zu lassen. Das folgende Dokument ist Ausdruck eines behutsamen Anpassungsprozesses für die Zukunft.

\section{Lebenswelten gestalten und Komplexität begegnen}

Im Vergleich zu der vor fünf Jahren erstmals publizierten Version des Fachdidaktischen Grundkonsenses kommt es in der überarbeiten Fassung zu einer Nachschärfung des Konzepts der ökonomischen Allgemeinbildung für das Fach GW. Zentrales Ziel ist dabei, Jugendliche zu befähigen, kritisch und selbstbestimmt ihre vielfältigen Rollen in ökonomisch, sozial und politisch geprägten Lebenswelten (vgl. Fridrich \& Hofmann-Schneller 2017) zu reflektieren und zu gestalten. Darüber hinaus werden aktuelle Erkenntnisse aus Fachdidaktik und Bildungswissenschaft berücksichtigt, die auch in aktuellen Lehrplanänderungen zum Ausdruck kommen. Beispielhaft dafür steht die Orientierung an ökonomischen und geographischen Basiskonzepten als fachliche Ergänzung zur Kompetenzorientierung.

Unterricht, der am fachdidaktischen Grundkonsens orientiert ist, will die Entwicklung von Lernenden zu kritischen und mündigen Gestalterinnen und Gestaltern ihrer vielfältigen (räumlichen, gesellschaftlichen, politischen, ökonomischen und kulturellen) Umwelten unterstützen. Erst durch die Umsetzung dieses Anspruchs werden aktuelle Herausforderungen wie postfaktische Beliebigkeit, Superkomplexität (Barnett 2004) und multiple Krisenhaftigkeit im Unterricht erfahrbar, verhandelbar sowie als veränderbar erkannt (vgl. Bergmeister, Pichler \& Hintermann 2017).

\section{Theoriegeleitet entscheiden und handeln}

Der Grundkonsens signalisiert weiterhin deutlich die zentrale Bedeutung fachdidaktischer Handlungs- und Begründungsfähigkeit für (zukünftige) Lehrpersonen 
und bekennt sich zu den Prinzipien der Schüler/innenorientierung, Lebensweltorientierung, Zukunftsund Gegenwartsorientierung. Unverändert gehen wir davon aus, dass es nie nur eine adäquate Fachdidaktik GW geben kann, sondern komplexe soziale und ökonomische Phänomene auch verlangen, fachdidaktische Forschung in Hinblick auf Mehrperspektivität und Kontingenz auszurichten. Unabdingbar scheint hierfür die Berücksichtigung des Pluralismus der Forschungs- und Lehrparadigmen, um einen differenzierten Rahmen für einen individuell begründbaren GW-Unterricht zu schaffen. Dieser Anspruch verpflichtet zukünftige Lehrpersonen dazu, sich im Rahmen ihrer Ausbildung mit den im Fachdidaktischen Grundkonsens zusammengefassten inhaltlichen und fachdidaktischen Konzepten und Modellen auseinander zu setzen. Auf dieser Basis sind Entscheidungen im Planungsprozess von Lehr- und Lernprozessen im Geographie- und Wirtschaftskunde-Unterricht der Sekundarstufe zu begründen. Bereits im Beruf stehende Lehrkräfte sind aufgefordert, durch laufende Fortbildung ihr fachdidaktisches Repertoire auf den jeweils aktuellen Erkenntnisstand zu bringen.

\section{Lehre und Unterricht in GW weiterentwickeln}

Wir bedanken uns bei allen Kolleg/innen, die sich am Diskussionsprozess beteiligt haben und sich darüber verständigt haben, diesen gemeinsamen Anspruch in Unterricht, Lehre und Forschung in der Verbundregion Nordost umzusetzen.
Mit der Veröffentlichung des Grundkonsenses vor fünf Jahren haben wir die Hoffnung formuliert, die Diskussion über den Kern der fachdidaktischen Bildung im Fachbereich Geographie und Wirtschaftskunde ein Stück weit vorangetrieben zu haben und damit zur österreichweiten Auseinandersetzung anzuregen. Diesen Wunsch bekräftigen wir im Zuge des Relaunches. Entwickeln wir gemeinsam Geographie und Wirtschaftskunde weiter! Der Fachdidaktische Grundkonsens möge durch die Kürze und Prägnanz seiner Formulierung Lehrende und Lernende weiterhin inspirieren und ein belastbarer Trittstein der Entwicklung von Bildungsprozessen in Geographie und Wirtschaftskunde werden.

\section{Literatur}

Barnett, R. (2004): Learning for an Unknown Future. Higher Education Research and Development. 23(3), 247-260.

Bergmeister, F., H. Pichler \& Ch. Hintermann (2017): Perspektiven eines zukunftsfähigen GW-Unterrichts 2.0 in Zeiten von Krisendiskurs, Superkomplexität, Postfaktizität und Kontingenz. In: ÖGL 61/1 (in Druck).

FridrichCh.\&M.Hofmann-Schneller(2017):Positionspapier „Sozioökonomische Bildung“. In: GW-Unterricht 145, 54-57. http://hw.oeaw.ac.at/0xc1aa500e_0x003574c2.pdf

Pichler, H. \& Ch. Vielhaber (2012): Der fachdidaktische Grundkonsens am Institut für Geographie und Regionalforschung - eine zukunftsfähige Orientierungshilfe? In: GW-Unterricht 128, 45-46. http://www.gw-unterricht. at/images/pdf/gwu_128_045_046_pichler_vielhaber.pdf 


\section{Fachdidaktischer Grundkonsens \\ der Fachdidaktik-Lehrenden im Lehramtsstudium \\ Geographie und Wirtschaftskunde in der Verbundregion Nordost}

Lehr-/Lernprozesse im GW-Unterricht der Sekundarstufe sind immer (fach)didaktisch zu begründen! Das verpflichtet die Lehrenden, ausgehend von ausgewählten didaktischen Konzepten und Modellen, unterrichtsrelevante Inhalte und Methoden in Lehr-/Lernprozesse überzuführen. Hierfür ist auch die Konzeption der Lehrpläne zu analysieren und in konkreten Handlungsoptionen im Unterricht umzusetzen.

Der GW-Unterricht fokussiert auf Lebenswelten und die in gesellschaftlichen Bezügen räumlich und wirtschaftlich handelnden Menschen. Gemäß den Zielen der sozioökonomischen Bildung wird Wirtschaft als gesellschaftlich eingebettet und veränderbar verstanden. Lernende sollen ihre komplexen Lebenswelten im GW-Unterricht durch kontinuierliche Reflexion und Entwicklung der individuellen Orientierungs-, Urteils- und Handlungsfähigkeit als mitgestaltbar erfahren können. Dieses Anliegen umfasst die Bereiche Haushalt, Konsum, Arbeitswelt, Gesellschaft und Umwelt auf allen Maßstabsebenen.

Folgende didaktische Konzepte und Modelle gehören zum Standardprogramm der gemeinsam von Universität und Pädagogischen Hochschulen in der Verbundregion Nordost getragenen Lehramtsausbildung und damit zum Begründungswerkzeug für die fachdidaktische Ausrichtung des GW-Unterrichts:

- Curriculumtheoretische Didaktik

- Kritisch-Konstruktive Didaktik

- Lehr-Lerntheoretische Didaktik

- Konstruktivistische Didaktikansätze

Bei der Planung bzw. Inszenierung von Lehr-/Lernprozessen des GW-Unterrichts sollten aus den folgenden didaktischen und methodischen Prinzipien jene ausgewählt werden, auf die fallspezifisch Bezug genommen wird:

- Schüler/innenorientierung und Lebensweltorientierung

- Handlungsorientierung

- Aktualitätsorientierung und Zukunftsorientierung

- Orientierung am Prinzip des Exemplarischen

- Orientierung an geographischen und ökonomischen Basiskonzepten

- Orientierung an Qualifikationsansprüchen, die zur Lösung komplexer Problemstellungen befähigen (Kompetenzorientierung)

- Orientierung am Prinzip der inhaltlichen Mehrperspektivität und der Kontingenz

- Politische Bildung auf Basis gesellschaftskritischer Reflexionen

- Partizipative Unterrichtsmethoden, selbstbestimmtes, individualisiertes und kooperatives Lernen sowie methodische Vielfalt

Zumindest folgende unterschiedliche Raumkonzepte der Fachwissenschaft sollen im Rahmen von Lehr-/ Lernprozessen berücksichtigt werden:

- Raum als Container

- Raum als System von Lagebeziehungen und Reichweiten

- Wahrnehmungsraum

- Konstruierter Raum

Auf folgende zusätzliche Aspekte der Themenerschließung ist im GW-Unterricht zu achten:

- Wirklichkeitsnähe und Alltagstauglichkeit

- Konflikt- und Widerspruchspotenzial

- Kritische Medienkompetenz

- Geschlechtersensibilität und Diversität

- Transkulturalität

- Arbeitswelt- und Berufsorientierung 